\section{CNS Spectrums}

www.cambridge.org/cns

\section{Editorial}

Cite this article: Herrera-Ferrá K, Nicolini H, and Giordano J (2022). Professional attitudes toward the use of neuromodulatory technologies in Mexico: Insight for neuroethical considerations of cultural diversity. CNS Spectrums 27(3), 255-257. https://doi.org/10.1017/S1092852920002151

Received: 04 August 2020

Accepted: 25 November 2020

\section{Author for correspondence:}

*Karen Herrera-Ferrá,

Email: ferra_karen@hotmail.com

\title{
Professional attitudes toward the use of neuromodulatory technologies in Mexico: Insight for neuroethical considerations of cultural diversity
}

\begin{abstract}
Karen Herrera-Ferrá ${ }^{1,2 *}$ (1), Humberto Nicolini ${ }^{1,3}$ and James Giordano ${ }^{2,4}$
${ }^{1}$ Asociación Mexicana de Neuroética, Atizapán, State of Mexico, Mexico, ${ }^{2}$ Neuroethics Studies Program, Pellegrino Center for Clinical Bioethics, Georgetown University Medical Center, Washington, DC, USA, ${ }^{3}$ Lab. de Enfermedades Psiquiátricas, Neurodegenerativas y Adicciones, Instituto Nacional de Medicina Genómica (INMEGEN), Mexico City, Mexico, and ${ }^{4}$ Departments of Neurology and Biochemistry, Georgetown University Medical Center, Washington, DC, USA
\end{abstract}

Neurotechnological interventions for specific cognitive, emotional, and behavioral conditions have been developed and employed in many parts of the world, and the use of "the most current tools" to affect thought and behavior has been abundant through much of human history. Examples include trephinations (ie, Aztecs, Egyptians, Galen in Rome, Burckhardt in Switzerland), leucotomies (eg, Moniz in Portugal), transorbital lobotomies (eg, Freeman in the US), and electric stimulation (eg, Delgado in Spain). The use, misuse, and overuse of such techniques during the 1960s exposed significant medical and ethical concerns and controversies about their validity, safety, success, risks, harms, and value, thereby calling their further use into question.

In recent years, the increased global burden of mental disorders, and the major efforts and advanced resources toward advancing neuroscience and neurotechnology (neuroS/T) from major brain initiatives from developed countries, ${ }^{1}$ have prompted, at very least (1) a re-evaluation of currently available approaches; and (2) development of newly emerging methods for assessing and affecting brain structure and functions. Neuromodulatory approaches have been-and are being further-developed to increase the sophistication, capability, accuracy, and effectiveness of both transcranial interventions (ie, transcranial electrical stimulationtES; transcranial magnetic stimulation-TMS; vagal nerve stimulation-VNS) as well as more invasive approaches (eg, indwelling VNS; deep brain stimulation-DBS). Although these techniques and tools are mainly for therapeutic aims, TMS and tES are also currently utilized for optimizing performance (viz. enhancement). ${ }^{2}$ Acknowledging the potential scope and impact of enhancement on decision-making (ie, nudging by Thaler, ${ }^{3}$ and manipulation of cognitive biases by Kahneman ${ }^{4}$ ), has increased realistic concerns about the use of neuroenhancement in the social domain. ${ }^{5}$

Thus, the rapid pace of development, and increasing clinical and social demand for these neuromodulatory technologies give rise to ethical, legal and social issues, question, problems, and caveats that need to be addressed, especially because these neurotechnologies are not always subject to rigorous regulatory approval process before becoming available to the medical and general public. ${ }^{6}$ To illustrate, in the United States (US), DBS can be employed under the "Humanitarian Device Exemption" issued by the Food and Drug Administration (FDA), which requires ongoing detailed scrutiny of the potential promises, benefits, risks, and harms for prudent and responsible translational clinical use. ${ }^{7}$ However, such iterative analysis and oversight do not regard or regulate transnational and cross-cultural implications when this neurotechnology is considered and/or applied for medical (and/or non-medical) uses in countries with different ecologies (ie, culture, economic resources, politics, philosophies, ethics, and laws), such as Mexico.

Recently, we explored mental health clinicians' and researchers' perceptions and concerns regarding the use of neuromodulatory techniques in Mexico, as compared to those reported in the international literature, ${ }^{8}$ and if there are also specific local neuroethical, legal, socio-cultural issues (NELSCI) relevant to such distinctions or similarities. ${ }^{9}$

We found that the majority of the respondents who utilize neuromodulatory techniques are professionally trained, and employed various forms of neuromodulatory approaches for treatment of psychiatric disorders (eg, depression and OCD), as consistent with those approved by the US FDA, and Parkinson's disease and other movement disorders, with such approaches regarded to be generally effective $(60 \%)$ and safe $(81 \%)$.

The preferred neuromodulatory techniques used in clinical practice and/or research areas were TMS and DBS; similar to other countries. Interestingly, tES, although somewhat more affordable and accessible than TMS, was used to a lesser extent, perhaps due to a paucity of both protocols and evidence of positive results. TMS and DBS use in research is notably limited (which 
differs from such use in developed countries), due to economic limitations, and inconsistent and slow oversight by ethics committees; which has fostered the use of external institutional review board (IRBs) that are expensive and thus, limited to the few wellfunded protocols.

Issues of informed consent, distributive justice, concerns about alteration of personality and authenticity, enhancement, stigma, and unknown side effects were regarded in priorities and ways similar to those reported in the international literature. ${ }^{8}$ But other considerations, such as violation of patients' dignity and rights, lack of dialogue between peers, experts, and patients relevant to stakeand shareholders' values and influence, and in each and all of these contexts, an absence of concern about and/or explicit address of cultural variables, all require further exploration and engagement within the mental health community in Mexico. To be sure, many of these issues are at the intersection of ethics and law, which prompts questions about differences-and problems-in legal and policy regulation of current and emerging neuroS/T in Mexico, implications for research and medical tourism, and relevance to international influence of-and upon-neuroS/T development and use in Mexico within contexts and circumstances of global economic, and hegemonic trends.

In this light, we found issues of regulation, enforcement, compliance, and monitoring (RECM) were regarded as a significant concern (although-and perhaps because-RECM are not legal requirements in Mexico). The RECM gap (which is not exclusive to Mexico) has been discussed in relation to other "well-being neuroenhancements" (eg, vitamins and herbalism), and these interventions are not labeled as "treatment products"; but there is misleading information regarding their use, safety, effectiveness, interactions with other substances, and risk of medical complications and adverse side effects. Both neuromodulatory techniques and other "well-being neuroenhancements" are used outside the clinical, fostering further concerns about RECM and derivative neuroethical issues (ie, autonomy, limits of enhancement, effects upon personality, civic readiness, etc.), which are similar to those reported in the developed world. ${ }^{8,10,11}$ Despite such concerns about RECM, there is little academic engagement of these issues in the training of researchers and clinicians. This too may reflect poor or non-existent funding of ethics/bioethics/neuroethics studies and programs. ${ }^{12,13}$

As well, we have described a general lack of receptivity for the use of such neuroS/T in Mexico, due, in part, to the persistence of a cosmogenic Pre-Hispanic vision of the "nature" of the human being that has resisted changes incurred by other anthropologies (ie, Western philosophy and medicine)., ${ }^{9,14}$

Such cultural considerations also influence perceptions about (unknown and/or perhaps as yet "unknowable," transcendent) effects of neuroS/T. Thus, any meaningful discourse about development and use of such approaches must also address the importance of cultural competence (in clinical and research professionals, as well as corporate entities providing neuroS/T within Mexico). ${ }^{15-18}$

This is of particular note, given that $90 \%$ of global neuropsychiatric research is performed in the $10 \%$ of the developed world's mental health population ${ }^{19}$ and hence, a significant proportion of global developing populations and ecologies are inadequately represented. This limited representation of ethnodiversity constitutes an important knowledge gap with respect to specific cultural variables (ie, beliefs, values, needs, traditions, and perceptions) that could shape attitudes toward the use-or non-use-of neuroS/ $\mathrm{T},{ }^{9,20,21}$ which could mislead both global translational safety and effectiveness, and neuroethico-legal and sociocultural frameworks that address and guide such issues in practice. To mitigate or prevent such inadequacies, we advocate a complementary and proactive integration of transnational and cross-cultural analysis to (1) identify local NELSCI and influences in and for neuromodulatory techniques; (2) improve global translational and transnational safety, viability, compatibility, and value, and (3) improve efforts to enhance global benefits of neuroS/T research and applications of emerging developments in practice. ${ }^{22}$ We believe that ongoing efforts toward these goals are both important and necessary if neuroS/T-and neuroethics - are to be relevant, applicable, and meaningful to current and near-future global value.

Funding. Profs. Karen Herrera-Ferrá and Humberto Nicolini received no specific grant from any funding agency, commercial or not-for-profit sectors Prof. James Giordano's work was supported in part by funding from the Henry Jackson Foundation for Military Medicine; Leadership Initiatives; BNB International; NeuroGen; the Creighton University Visiting Professorship; Coburg University Distinguished Visiting Professorship in Integrative Health Promotions; and federal funds 2UL1TR001409-06 from the National Center for Advancing Translational Sciences (NCATS), National Institutes of Health, through the Clinical and Translational Science Awards (CTSA) Program, a trademark of the US Department of Health and Human Services, part of the Roadmap Initiative, "Re-Engineering the Clinical Research Enterprise."

Disclosures. Karen Herrera-Ferrá, Humberto Nicolini, and James Giordano do not have anything to disclose.

\section{References}

1. International Brain Initiative (IBI). International Brain Initiative. International Brain Initiative; 2018. Accessed December 2019. https://www. internationalbraininitiative.org/about-us.

2. Dresler M, Sandberg A, Bublitz C, et al. Hacking the brain: dimensions of cognitive enhancement. ACS Chem Neurosci. 2019;10(3):1137-1148. doi: 10.1021/acschemneuro.8b00571.

3. Thaler RH. Nudge: Improving Decisions About Health, Wealth, and Happiness. New Haven, Connecticut: Yale University Press; 2008.

4. Kahneman D. Thinking, fast and slow. New York, NY: Farrar, Straus and Giroux; 2011.

5. Shook JR, Giordano J. Neuroethics beyond normal. Performance enablement and self transformative technologies. Camb Q Health Care EthicsNeuroethics. 2016;25(121):140

6. Shepherd RK, Villalobos J, Burns O, et al. The development of neural stimulators: a review of preclinical safety and efficacy studies. $J$ Neural Eng. 2018;15(4):041004. doi: 10.1088/1741-2552/aac43c.

7. Fins JJ, Mayber HS Misuse of the FDA's humanitarian device exemption in deep brain stimulation for obsessive-compulsive disorder. Health Affairs. 2011;30(2):302-311. doi:10.1377/hlthaff.2010.0157.

8. Zuk P, Torgerson L, Sierra-Mercado D, et al. Neuroethics of neuromodulation: an update. Curr Opin Biomed Eng. 2018;8:45-50. doi: 10.1016/j. cobme.2018.10.003.

9. Herrera-Ferrá K, Saruwatari-Zavala G, Nicolini-Sánchez H, et al. Neuroética en México: Reflexiones médicas, legales y socioculturales. Bioethics Update. 2019:89-106. doi:10.1016/j.bioet.2019.05.001.

10. Williams NR, Taylor JJ, Snipes JM, et al. Interventional psychiatry: how should psychiatric educators incorporate neuromodulation into training? Acad Psychiatry. 2014;38(2):168-176. doi:10.1007/s40596-014-0050-x.

11. Shook JR, Giordano J. Moral bioenhancement for social welfare: are civic institutions ready? Front Sociol. 2017;2(21):1-5.

12. CONBIOÉTICA. Educación en Bioética. Gaceta Conbioética. 2016;5(20). https://www.gob.mx/cms/uploads/attachment/file/361044/Gaceta_20_ Educacion.pdf.

13. Pronunciamiento en torno a la educación. Compromiso IMSS-UNAM. 2018. https://www.gob.mx/cms/uploads/attachment/file/470834/6._Compromiso_ IMSS_UNAM_2018_completo.pdf. Accessed October 2020.

14. Comisión Nacional Para el Desarrollo de los Pueblos Indígenas (CDI). Consulta sobre mecanismos para la protección de los conocimientos tradicionales, expresiones culturales, recursos naturales, biológicos y genéticos de 
los pueblos indígenas. Mexico City: Comisión Nacional para el Desarrollo de los Pueblos Indígenas; 2011. https://www.gob.mx/cms/uploads/attach ment/file/37014/cdi_consulta_proteccion_conocimientos_tradicionales.pdf. Accessed October 2020.

15. Rattani A, Hyder AA. Developing an Islamic research ethics framework. $J$ Religion Health. 2019;58:74-86.

16. Saadat SH. Improving trend of adhering to ethical measures in Iranian research in human genetics: a survey from 2005 to 2009; and the road ahead. Iran J Publ Health. 2013;42(10):1181-1186.

17. Sherman M. Balancing indigenous principles and institutional research guidelines for informed consent: a case study from the Peruvian Amazon. AJOB. 2012;3(4):53-68.
18. Marshall PA. Indigenous populations: decision making and informed consent to research. AJOB Primary Res. 2012;3(4):69-71.

19. Stein D, Giordano J. Global mental health and neuroethics. BMC Med. 2015;13(44). https://doi.org/10.1186/s12916-015-0274-y.

20. Herrera-Ferrá K. Global mental health and the treatment gap: a human rights and neuroethics concern. In Dan Stein and Ilina Singh, Global Mental Health and Neuroethics. London: Elseiver; 2020:133-143.

21. Shook J, Giordano J. A principled and cospmopolitan neuroethics: consideration for international relevance. Philos Ethics Humanit Med. 2014;9(1). https://doi.org/10.1186/1747-5341-9-1.

22. Shook J, Giordano J. Toward a new neuroethics in a multipolar and multicultural world. Global-E. 2020;13(56):2-10. 